\title{
MEASUREMENTS OF TERMINAL VELOCITIES OF CIRRUS CLOUDS IN THE UPPER TROSPHERE
}

\author{
Jan Bai Nee, W. N. Chen, C. W. Chiang, S.K. Das \\ Department of Physics, National Central University, Chungli, Taiwan 32001 \\ jbnee@phy.ncu.edu.tw
}

\begin{abstract}
Cirrus clouds are composed of ice crystals condensed from humidity due to low temperature condition in the upper atmosphere. The microphysics of cirrus clouds including sizes and shapes of ice particles are not well understood but are important in climate modeling. Ice crystal will fall under gravitational sedimentation to reach terminal velocities which depend on the size, mass, and ice habit. We studied here the terminal velocity of cirrus clouds by using lidar observations at Chungli $(25 \mathrm{~N}, 121 \mathrm{E})$. The terminal velocities for a few cases of stable cirrus clouds are measured to determine the ice particle sizes and processes in the upper atmosphere.
\end{abstract}

\section{INTRODUCTION}

Cirrus cloud is an important element in understanding the climate and radiation of the earth's atmosphere. However, modeling efforts are hampered by the lack of many important parameters which include ice particle size, habit, terminal velocity, composition and optical properties and related parameters. Terminal velocity is caused by the gravitational settling of ice crystals and depends on the size, mass, and shape of the ice crystals. Terminal velocity is important not only for understanding the microphysics of cirrus clouds but also for understanding the dehydration process in the upper tropospheric (UT) region.

In this paper, we study, terminal velocity by using lidar observations at Chungli (25 N, 121E), Taiwan. As previously reported, cirrus clouds in the UT are often observed [1]. For stable clouds, the terminal velocities can be measured and used for deriving the ice crystal properties [1-2].

\section{Instrument and methodology: Lidar system for cirrus research}

The lidar facility used to detect the cirrus cloud has been reported before [1-2]. Briefly, a laser at $532 \mathrm{~nm}$ operated at $30 \mathrm{~Hz}$ frequency is sent into the atmosphere in the zenith direction. A Newtonian type telescope of 18 inch diameter is employed for the collection of scattering light. The backscattering light is detected by a photomultiplier tube combined with an interference filter centered at $532 \mathrm{~nm}$ with FWHM $1 \mathrm{~nm}$. The photon counting signals are analyzed by a fast multichannel analyzer (SR430) and stored in a computer. Polarization measurements are carried out also to derive particle nature. The lidar signals are analyzed according to the Fernald integration method in terms of backscattering and depolarization ratios. For normal cirrus clouds, the average backscattering ratio is about 10 and depolarization ratio $<0.5$.

\section{Observation of falling cirrus clouds}

Cirrus clouds are often observed in the upper troposphere. Factors affecting the height, composition, and stability of cirrus cloud include wind, waves, temperature, and transport of humidity. However, descending stable cirrus clouds are frequently observed. Figures (1a) and (1b) show the contours for a cirrus clouds at about $16 \mathrm{~km}$ in the tropopause region. Fig. (1a) shows results for the backscattering ratio (BR) and Fig (1b) for the depolarization ratio (DR). As we can see in Figure 1, falling cirrus cloud is observed in both plots and terminal velocity can be measured as shown in Fig.2. The cloud position is defined by using a cloud centroid position given as: $h=\int \mathrm{Rzdz} / \int \mathrm{Rdz}$ where $\mathrm{R}$ is the backscattering ratio and $\mathrm{z}$ is the height position. Fig. 2 shows the centroid of the cloud descended $0.4 \mathrm{~km}$ in about 5 hours corresponding to a speed about $2.5 \mathrm{~cm} / \mathrm{sec}$.

Fig. 3 shows a case of rising and lowering of high cloud observed. In the top panel, we observe 
the rising of cirrus cloud and a growth process. The process will freezes out the humidity and causes dehydration in the region. The upper plot of Fig 3 gives a slow rising speed of $3 \mathrm{~cm} / \mathrm{s}$ and the bottom part shows varied speed depending on the cloud positions. We can see the cloud are separated into three parts each descending with different speeds ranging between 6 to $18 \mathrm{~cm} / \mathrm{sec}$ depending on the height. By assuming ice particles shapes such as hexagonal plate and cylinder, the speeds can be determined.
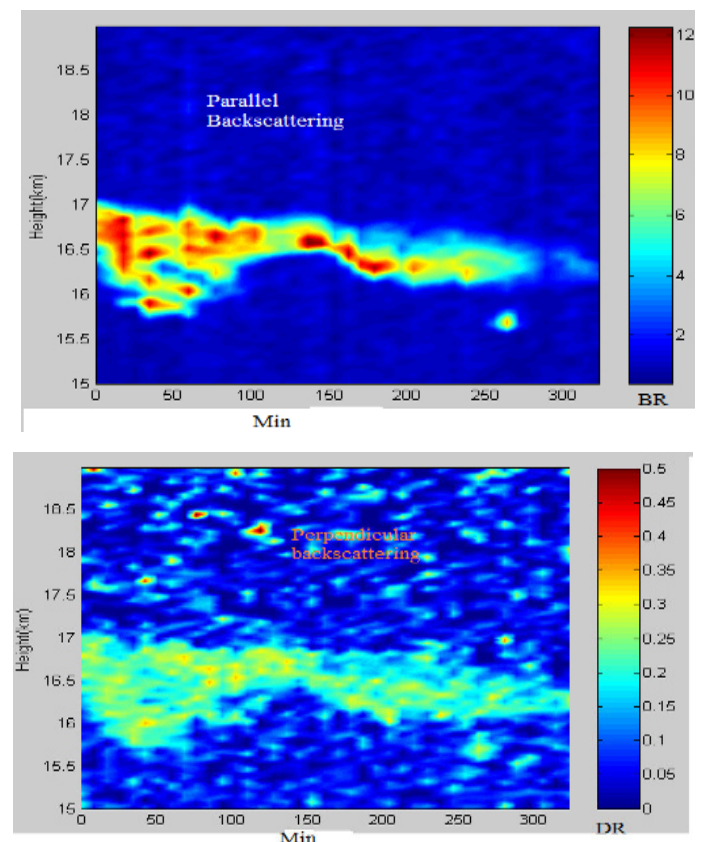

Figure 1. Contour diagram of a $16 \mathrm{~km}$ cirrus clouds observed on 2004 June 29. (1a) top: backscattering ratio(BR) for a cirrus cloud at 16 $\mathrm{km}(\mathrm{lb})$ bottom: the depolarization ratio (DR).

\section{Summary}

Terminal velocity of cirrus clouds can be measured by using lidar observations. A few cases of high cirrus cloud showing steadily descending are presented here. We found terminal velocities of clouds ranged $2-20 \mathrm{~cm} / \mathrm{s}$ corresponding to various ice crystals of sizes. Based on ice habit model, terminal velocity can be used to derive sizes of different types of ices. For hexagonal plate and cylinder, the size of ices of $1-100 \mu \mathrm{m}$ are determined as will be shown.

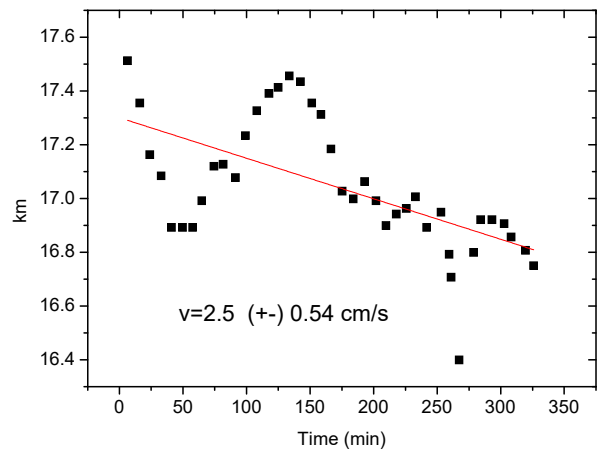

Figure 2. The cloud centroid position as a function of time.
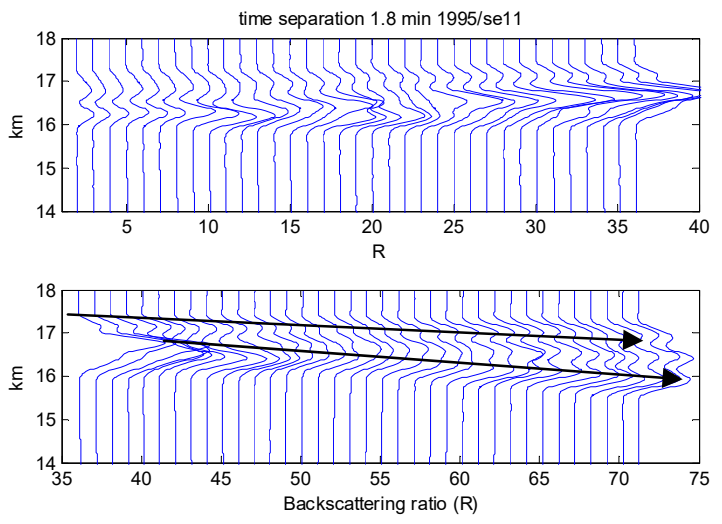

Figure 3. A case of tropopause thin cirrus ascending (upper plot) and descending (lower plot). The profiles are backscattering ratio $\mathrm{R}$ progressively offset by a constant in $1.8 \mathrm{~min}$ time interval.

\section{REFERENCES}

[1] Nee,J. B., W. N. Chen, C. N. Lien, C. I . Lin, 1998: Lidar detection of cirrus cloud in Chung-Li (25 N, 121 E) , J. Atmos. Sci.55, 2249-2257.

[2] Das, S.K., C.-W. Chiang, and J. B. Nee, , 2009: Characteristics of cirrus cloud and its radiative properties based on lidar observation over ChungLi, Taiwan, Atmos. Res., 93,723-735.

[3] Das, S. K., J. B. Nee, and C. W. Chiang, A LIDAR study of the effective size of cirrus ice crystals over Chung-Li, Taiwan, J. Atmos. Sol. Terr. Phys. 72,781-788, 2010. 